\title{
Multimorbidity in a large district hospital: A descriptive cross-sectional study
}

\author{
S Roche, ${ }^{1}$ 4th-year medical student; E de Vries, ${ }^{2}$ MB ChB, MFamMed, FCFP (SA) \\ ${ }^{1}$ Faculty of Health Sciences, University of Cape Town, South Africa \\ ${ }^{2}$ School of Public Health and Family Medicine, Faculty of Health Sciences, University of Cape Town, South Africa; \\ Mitchell's Plain District Hospital, Western Cape Department of Health, Cape Town, South Africa
}

Corresponding author: E de Vries (elma.devries@westerncape.gov.za)

\begin{abstract}
Background. There is substantial research documenting the burden of disease globally and in the South African (SA) primary care context. Few studies address the disease profile and its implications in the SA hospital setting.

Objectives. To describe the disease profile in the internal medicine department of a large district hospital, using variables related to comorbidity and patient length of stay. The study included specific exploration into the HIV/tuberculosis (TB) syndemic, the acuity of HIV disease, and lifestyle risk factors.

Methods. The sample population consisted of all consecutive admissions to the internal medicine department of a large district hospital in the Cape metropole during May 2015. A retrospective folder review and subsequent data analysis were completed.

Results. Hypertension, HIV, type 2 diabetes mellitus, TB and cardiac failure were the five most prevalent diseases. Extensive multimorbidity was observed, with $86.0 \%$ of patients suffering from two or more diseases concurrently. The average number of comorbidities per patient was 3.4, although no clear relationship between the number of comorbidities and length of stay was found. Of the various diseases, only TB and HIV were associated with above-average length of stay, particularly among co-infected patients and those who had defaulted from or never received antiretroviral (ARV) treatment. Compared with patients currently receiving ARVs, much higher proportions of patients who had defaulted from or never received ARV treatment had CD4+ counts $<200$ cells/ $\mu \mathrm{L}$. Of the lifestyle risk factors investigated, a history of excessive alcohol use and/or drug use was associated with an increased length of stay. Most patients were discharged home, with $15.7 \%$ being referred to other institutions.

Conclusions. Chronic conditions, particularly HIV, TB and non-communicable diseases, represented much of the disease profile in the internal medicine department. Of the comorbidities investigated, the greatest contributor to length of stay was HIV/TB co-infection. Factors such as HIV, TB and substance use that increase length of stay cannot be impacted upon by the district hospital staff in isolation. To improve the health of communities, we require partnerships between doctors, community health providers and patients with their families. Multimorbidity was widespread, suggesting the need to include an understanding of multimorbidity, including the patient perspective, in medical education and health system reform.
\end{abstract}

S Afr Med J 2017;107(12):1110-1115. DOI:10.7196/SAMJ.2017.v107i12.12397

The global burden of disease changes constantly as economic developments and health advances influence disease processes and human lifestyles. As life expectancy increases, especially in developing countries, there is a shift from a burden of acute infectious diseases to one of chronic infectious diseases and non-communicable diseases (NCDs). ${ }^{[1]}$

Treatment is becoming more accessible for populations in the developing countries, especially treatment of HIV with antiretrovirals (ARVs). ${ }^{[1]}$ With reduced mortality, the HIV/tuberculosis (TB) syndemic has risen alongside NCDs associated with urbanisation. ${ }^{[2]}$ This rise in HIV prevalence has been associated not only with an increased incidence of opportunistic infections ${ }^{[3]}$ but also with increased risks of developing epilepsy, renal disease, heart disease, chronic obstructive pulmonary disease and many other comorbidities. ${ }^{[4]}$ South African (SA) studies have also demonstrated that ARVs are an independent risk factor for cardiometabolic disease. ${ }^{[5,6]}$

The rising burden of NCDs has also been linked to increasing exposure to environmental and behavioural risk factors. ${ }^{[7]}$ Rapid urbanisation has been associated with an increased prevalence of type 2 diabetes mellitus in low- to middle-income countries. ${ }^{[4]}$ In 2012, the Western Cape Mortality Profile in SA showed that the five most common causes of death among females were diabetes, cerebrovascular disease, ischaemic heart disease, HIV and TB. ${ }^{[8]}$ A similar burden of fatal disease was described for men, with interpersonal violence replacing diabetes mellitus. ${ }^{[8]}$

This growing burden of disease has been demonstrated in many studies performed at a primary care level, documenting a high prevalence of NCDs and extensive multimorbidity in outpatients. Barnett et al..$^{[9]}$ describe multimorbidity as the norm among patients, a statement with many public health implications. This same study found a greater prevalence of multimorbidity in the most economically deprived population groups, and at a younger age, than in more affluent groups. ${ }^{[9]} \mathrm{A}$ recent study ${ }^{[6]}$ performed in an informal settlement in Cape Town, SA, confirmed these findings, highlighting in particular the frequent comorbidity of hypertension and diabetes mellitus. Notably, comorbidity associations were found not only between diseases of lifestyle, but also between infectious diseases such as TB and various NCDs. ${ }^{[6]}$ Mental illness is also associated with chronic disease, both infectious and non-infectious, and is particularly prevalent in those suffering from HIV and TB. ${ }^{[1,2]}$ Mental illness contributes to the patient's burden of multimorbidity, but also specifically affects quality of life and treatment outcomes. 
Despite extensive analysis of morbidity patterns at a population and primary care level, there is limited research into the disease profile at a district level of care in SA. Understanding multimorbidity in each healthcare context is essential, as patients with comorbid conditions are particularly vulnerable to suboptimal care. ${ }^{[10]}$ Multimorbidity in itself is an independent predictor of treatment complications and poor quality of life, and must therefore be thoroughly understood when making both clinical and institutional health decisions. ${ }^{[10]}$

\section{Objectives}

By investigating the disease profile at a district-level hospital, to add to the existing knowledge and understanding of multimorbidity in SA. In addition, the study aimed to specifically explore the HIV/TB syndemic, the acuity of HIV disease, lifestyle risk factors and patient length of stay.

\section{Methods}

Ethics approval was obtained from the Human Research Ethics Committee of the University of Cape Town (ref. no. 423/2015). This was a retrospective folder review. Admissions to the internal medicine department at a large district hospital in Cape Town were sampled, using consecutive admissions for the month of May 2015. Non-discharged and deceased patients were excluded, owing to inaccessibility of their folders. Each patient's folder number, age, date of birth, gender, triage details, comorbidities, lifestyle risk factors, length of stay and discharge destination were recorded.

Triage values consisted of each patient's triage code, determined by clinical discriminators that indicate acuity upon admission as per the South African Triage Score. ${ }^{[11]}$ A history of cigarette smoking, excessive alcohol use and drug use were the three lifestyle factors recorded. Length of stay was calculated in units of 24 hours. The discharge destination was either home or the referral institution. The CD4+ counts for HIV-positive patients were also recorded.

\section{Statistical analysis}

The data were analysed using Microsoft Excel, 2011 version (Microsoft, USA). The 10 most prevalent diseases and the prevalences of the various lifestyle risk factors were calculated. These diseases and lifestyle risk factors were then described in relation to patient length of stay and number of comorbidities, with a focus on HIV-positive patients and HIV/TB co-infected patients.

\section{Results \\ Sample population}

The sample population consisted of 491 patients from the internal medicine department, of whom $8.8 \%$ were readmitted and discharged during the same month. Of the patients, $57.4 \%$ were female and $42.6 \%$ male, with a mean age of 49.0 years (Table 1 ).

\section{Triage}

Of the patients, 38/491 (7.7\%) were coded green on admission, 130 (26.5\%) yellow, $231(47.0 \%)$ orange and $51(10.4 \%)$ red (the triage code was not documented for 41 patients). The most common triage code was therefore orange, with red and orange codes constituting $>50 \%$ of admissions.

\section{Prevalence of various diseases and lifestyle risk factors}

Hypertension (40.9\%), HIV (29.7\%) and type 2 diabetes mellitus (25.9\%) were the three most prevalent diseases, followed by current TB and cardiac failure (Table 2). HIV/TB co-infection was found in $17.1 \%$ of admissions. Mental illnesses counted as individual

\begin{tabular}{ll} 
Table 1. Characteristics of study participants \\
\hline Category \\
\hline Patients/folders \\
$\quad$ Patients in May discharge list, $N$ & 526 \\
$\quad$ Unavailable patient folders, $n$ & 35 \\
$\quad$ Total accessible folders, $n$ & 491 \\
$\quad$ Readmissions, $n(\%)^{*}$ & $43(8.8)$ \\
Gender, $n(\%)^{*}$ & \\
$\quad$ Female & $282(57.4)$ \\
$\quad$ Male & $209(42.6)$ \\
Age (years) & \\
$\quad$ Mean (range) & $49.0(13-88)$ \\
$\quad$ SD & 17.7 \\
$\quad$ Median (IQR) & $49.0(33.0-62.5)$ \\
SD $=$ standard deviation; IQR $=$ interquartile range. & \\
$\star \%$ of accessible folders. &
\end{tabular}

\section{Table 2. Prevalence of various diseases ${ }^{\star}(N=491)$}

\begin{tabular}{ll}
\hline Diagnosis & $\boldsymbol{n}(\%)$ \\
\hline Hypertension & $201(40.9)$ \\
HIV infection & $146(29.7)$ \\
Type 2 diabetes mellitus & $127(25.9)$ \\
Current TB & $108(22.0)$ \\
Cardiac failure & $75(15.3)$ \\
Pneumonia & $72(14.7)$ \\
Renal failure (acute and chronic) & $67(13.7)$ \\
COPD & $65(13.2)$ \\
Ischaemic heart disease & $62(12.6)$ \\
Asthma & $53(10.8)$ \\
Lower respiratory tract infection & $43(8.8)$ \\
Dyslipidaemia & $39(7.9)$ \\
Gastroenteritis & $38(7.7)$ \\
Cerebrovascular accident & $34(6.9)$ \\
Epilepsy & $32(6.5)$ \\
Anaemia & $28(5.7)$ \\
Sepsis & $21(4.3)$ \\
Cancer & $20(4.1)$ \\
Urinary tract infection & $20(4.1)$ \\
Delirium & $17(3.5)$ \\
Cor pulmonale & $15(3.1)$ \\
Deep vein thrombosis & $14(2.9)$ \\
Bronchiectasis & $13(2.6)$ \\
Dilated cardiomyopathy & $11(2.2)$ \\
TB tuberculosis; COPD $=$ chronic obstructive pulmonary disease. \\
${ }^{\text {Excluded if prevalence } \leq 2 \% .}$ \\
& \\
&
\end{tabular}

diagnoses, i.e. depression, bipolar mood disorder, anxiety disorder and schizophrenia, affected six or fewer patients each, but in the group as a whole, $6.3 \%$ of patients had mental illness.

When investigating the subcategories of TB patients, it was found that $66.7 \%$ suffered from pulmonary TB, the second and third most common forms being disseminated $\mathrm{TB}(16.7 \%)$ and multidrugresistant TB (10.2\%).

\section{Patient length of stay}

The average length of stay for all patients was 6.0 days. More than two-thirds of patients stayed for $\leq 6$ days, and the distribution of length-of-stay values showed that the mode was 3 days. 
Looking at the 10 most prevalent diseases, patients with current TB were found to have the greatest average length of stay ( 7.5 days), followed by HIV-positive patients (6.9 days). Patients with HIV/TB co-infection had an even greater average length of stay (7.7 days). No one of the remaining eight most prevalent diseases was associated with an average length of stay greater than that of the sample population.

Patients with HIV/TB co-infection were also found to have the highest proportion of individuals with an increased length of stay, with $39.3 \%$ of these patients staying $>8$ days (the 3 rd quartile) (Fig. 1). An increased length of stay was also seen in $>25 \%$ of patients with TB, HIV infection, renal failure and pneumonia (Fig. 1).

\section{Comorbidities of patients}

Of the patients, 427 suffered from more than one disease concurrently, putting the prevalence of multimorbidity at $87.0 \%$. The maximum number of confirmed diagnoses was 9 and the minimum was 1 , with a mode of 3 comorbidities. The average number of comorbidities for the sample population was 3.3 .

The highest number of comorbidities was found in patients with ischaemic heart disease, with an average of 4.8 concurrent diseases. Average length of stay was not associated with number of comorbidities, and for patients with a length of stay greater than the third quartile (length of stay $\geq 8$ days), the average number of comorbidities was also 3.3, no greater than that of the sample population.

\section{Contribution of HIV}

Investigation of the treatment status of HIV-positive patients showed that $44.5 \%$ of these patients were using ARVs during their hospital stay, $16.4 \%$ had defaulted from their ARV treatment before admission, and $39.0 \%$ were ARV naive upon admission.

Of the 146 HIV-positive patients, $88.4 \%$ had recorded CD4+ counts. The average CD4+ count was 218.3 cells/ $\mu \mathrm{L}$, with $62.8 \%$ of patients having a very low $\mathrm{CD} 4+$ count $(<200$ cells $/ \mu \mathrm{L})$. The proportion of patients with a very low $\mathrm{CD} 4+$ count was highest among those who had defaulted from ARV treatment (77.3\%), followed by those who had never received ARV treatment (70.8\%) and then those currently using ARVs (52.6\%) (Fig. 2).

Although CD4+ count was not directly associated with length of stay, average length of stay was found to be associated with ARV treatment status. Patients who had defaulted from ARV treatment had an average length of stay of 8.3 days, 2.5 days more than the average length of stay of patients using ARVs (Fig. 3).

\section{Contribution of lifestyle risk factors}

The most prevalent lifestyle risk factor was found to be cigarette smoking, with $34.8 \%$ of patients reporting a history of smoking, $16.7 \%$ excessive alcohol use and $7.7 \%$ drug use. Methamphetamines were the drugs most commonly used by these patients (46.3\%), followed by marijuana (31.7\%), mandrax (26.8\%) and heroin (4.9\%).

Investigation of the association between lifestyle risk factors and average length of stay showed that of the three lifestyle risk factors, drug abuse and excessive alcohol use were associated with an average length of stay greater than that of the sample population ( 7.7 days and 6.9 days, respectively) (Fig. 4). No significant association was shown between lifestyle risk factors and number of comorbidities.

\section{Discharge information}

Of the patients, $84.3 \%$ were discharged home. The remaining patients were referred to a level $2 / 3$ hospital $(6.7 \%)$, to a rehabilitation or

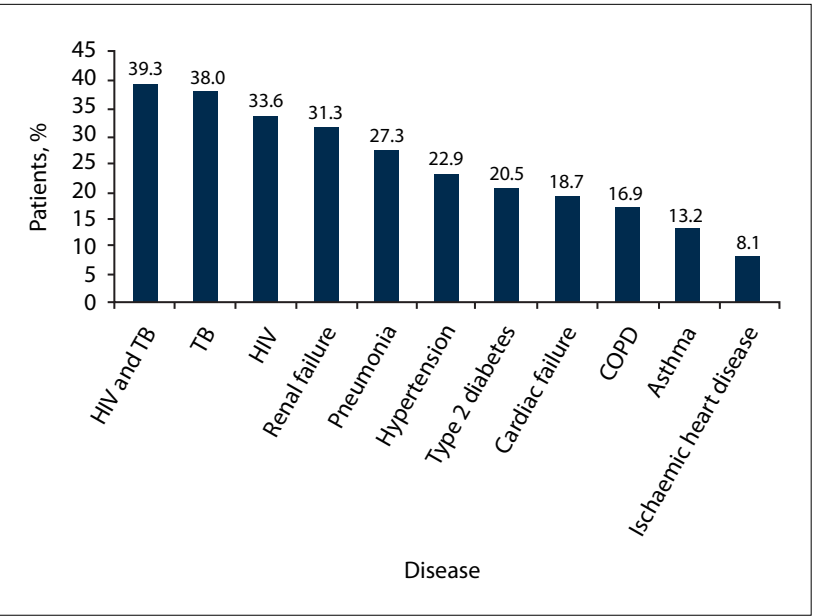

Fig. 1. Proportion of patients with length of stay $\geq 8$ days, comparing the 10 most prevalent diseases. $(T B=$ tuberculosis; $C O P D=$ chronic obstructive pulmonary disease.)

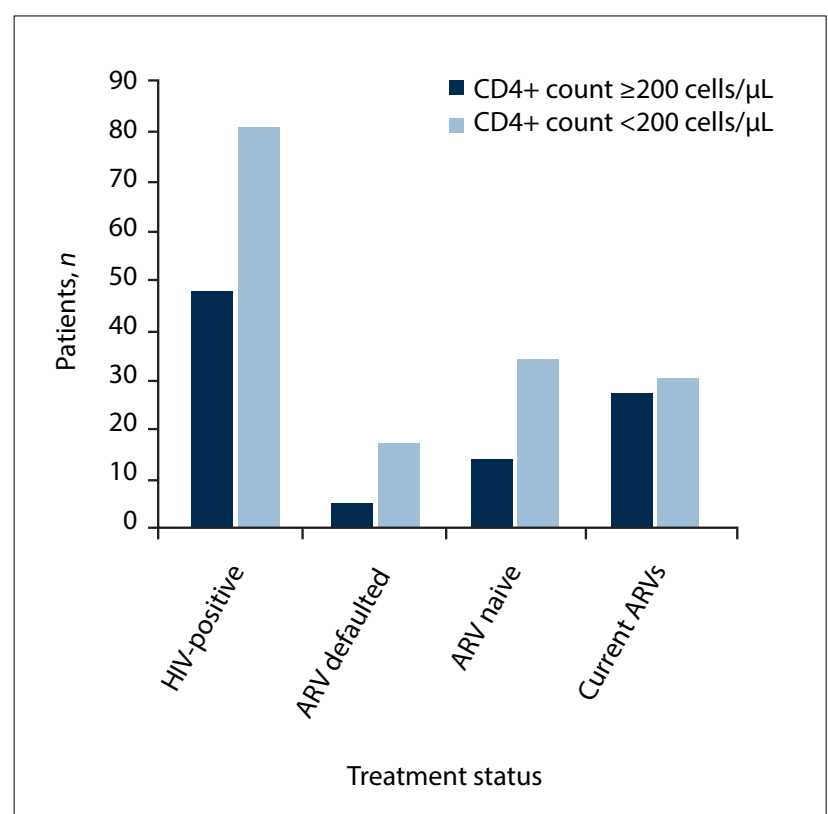

Fig. 2. CD4+ counts above and below 200 cells/ $\mu L$, according to ARV treatment status. $(A R V=$ antiretroviral. $)$

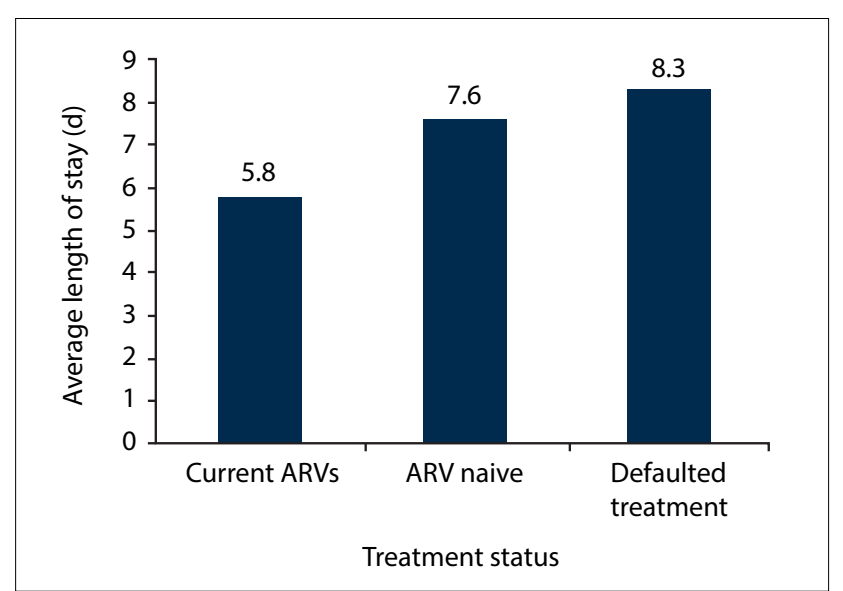

Fig. 3. Average length of stay of HIV-positive patients according to treatment status. 


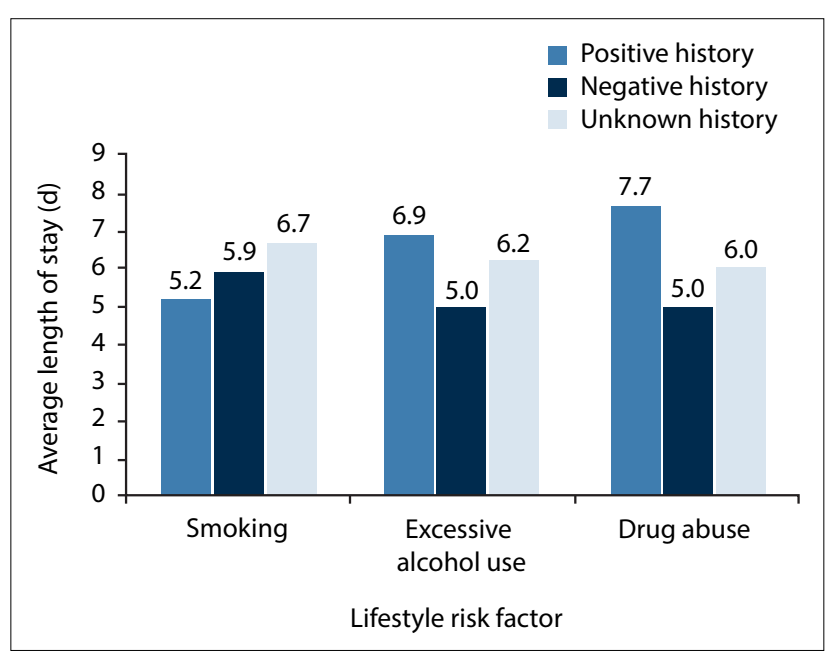

Fig. 4. Association between lifestyle risk factors and average length of stay.

intermediate-care facility $(4.7 \%)$ or to a specialised TB hospital (3.7\%).

\section{Discussion}

This study investigated the disease profile in the internal medicine department at a large district hospital in the Cape metropole during May 2015. This is a specific subset of the patient population that has decompensated to the extent of requiring admission to the hospital. While this study describes admissions to a district hospital, these need to be seen as part of the continuum of care ${ }^{[12]}$ from communitybased care and primary care through to referral to central hospitals. The clinical pressures of work may discourage busy doctors from thinking outside of the walls of the hospital, but it is important to have a health system perspective and consider ways of encouraging healthy behaviour after discharge. This is often left to primary care providers, despite hospital admission being an important opportunity for intervention. Training in brief behaviour change counselling on NCD lifestyle risk factors ${ }^{[13]}$ can improve the skills of both hospital and primary care doctors.

As expected, extensive comorbidity was observed throughout the sample population, with a high prevalence of both non-communicable and chronic infectious diseases. The results indicated that patients with HIV/TB co-infection or untreated HIV, those who had defaulted from ARV treatment, and those with a positive history of drug or excessive alcohol use in particular had an increased length of stay.

\section{Acuity of medical admissions}

Medical admissions were most frequently classified as urgent or very urgent, with more than half of the patients being classified as orange or red on admission according to the South African Triage Score. ${ }^{[11]}$

\section{Length of stay}

The average length of stay for all patients in the sample population was 6.0 days. This number is high compared with other district hospitals in SA, where the 2013/14 average length of stay in district hospitals was found to be 4.7 days nationally and 3.7 days in the Western Cape Province. ${ }^{[14]}$ According to departmental guidelines, a raised average length of stay may be linked to the decreased availability of hospital staff to oversee patient discharges. ${ }^{[14]}$ Delayed referral may also contribute to delayed discharge, suggesting that future research should incorporate data from the associated referral facilities. In the context of extensive multimorbidity, it is clear that patients may require multiple sites of care with an increased reliance on referral efficiency, making a well-connected multiprofessional team an important requirement for rapid patient turnover. ${ }^{[10]}$

In terms of the distribution of length of stay values, patients with HIV, TB or HIV/TB co-infection were the only ones with an average length of stay greater than that of the sample population. More than a third of these patients had a hospital stay of $>8$ days. A previous study at G F Jooste Hospital in Cape Town found an average length of stay of 9.6 days for inpatients with HIV. ${ }^{[3]}$ This suggests that although processes for generally improving patient turnover should be investigated, research can also be focused specifically on reducing in-hospital time of HIV, TB and co-infected patients, as well as avoiding admission by improving early diagnosis and treatment of HIV as outlined in the Joint United Nations Programme on HIV and AIDS (UNAIDS) 90-90-90 strategy. ${ }^{[15]}$

Average length of stay was also associated with various lifestyle factors. Both excessive alcohol use and drug use were associated with an average length of stay greater than the average for the sample population (Fig. 4). Doctors can play a role by providing brief behaviour change counselling ${ }^{[13]}$ and referring patients to community-based rehabilitation services.

\section{Multimorbidity}

The five most prevalent diseases were found to be hypertension, HIV, type 2 diabetes mellitus, TB and cardiac failure (Table 2), with HIV/TB co-infection in $17.1 \%$ of patients. Multimorbidity was recorded in $86.0 \%$ of patients and increased with patient age. These findings are consistent with various SA multimorbidity studies performed at a population and primary care level, suggesting that multimorbidity is an expected norm among patients at multiple levels of healthcare. ${ }^{[6,16,17]}$ This has vast implications for healthcare provision. Drug trials should be conducted with the knowledge of the most likely comorbidities and related treatments, ${ }^{[10]}$ medical education should teach an integrated approach instead of discussing diseases separately, ${ }^{[9]}$ and health professionals should be aware of the complexity of the challenges faced by a patient living with multiple comorbid conditions. ${ }^{[6]}$

This concept has been described by Shippee et al. ${ }^{[18]}$ who developed a patient-centred model of patient complexity. This model proposes that treatment adherence, healthcare utilisation and health outcomes are influenced by the relationship between the workload that is delegated to patients by healthcare providers (including managing self-care, treatment modalities, behaviour change and clinic visits), and patients' capacity to meet the demands of this workload (including physical and mental functioning, pre-existing health literacy and family and social support). In the Shippee model, multimorbidity is likely to both increase workload and reduce capacity - over time it adds complexity to patient experiences, and leads to ineffective and inappropriate outcomes. ${ }^{[19]}$ This model can assist doctors to be less judgemental and more patient centred when patients with multimorbidity find it difficult to adhere to treatment.

When patients are discharged, doctors are not always aware of their capacity to do what is expected of them. It is helpful to speak to the families when patients are discharged, as well as to refer to communitybased services. The importance of a good referral letter needs to be emphasised. When completed in full and with attention to detail, this can be an important tool in ensuring continuity of care. ${ }^{[12]}$

Interestingly, despite this issue of increasing complexity and poorer outcomes, we did not find multimorbidity to be associated with an increased length of stay in hospital. Patients with multiple diseases may have more complex management and require more resources 
while in hospital, but patients with comorbid conditions do not necessarily require care in the internal medicine wards for a longer period of time than those without.

Comorbidity was also not associated with any specific disease. Although ischaemic heart disease was associated with the greatest number of comorbidities, there was very limited variation across diseases. This suggests that other factors play a role in multimorbidity. In terms of the lifestyle risk factors investigated in this study, no significant relationship was found between any of the three lifestyle risk factors and number of comorbidities, despite previous research showing that smoking is a risk factor for multimorbidity. ${ }^{[16]}$

Other SA studies have described the role of socioeconomic factors in determining the extent of multimorbidity, suggesting that economic factors such as unemployment may underlie and lead to multimorbidity. ${ }^{[16]}$ The burden of multimorbidity is generally higher in the poor, who often have a reduced capacity to deal with ill health. ${ }^{[19]}$

Over $6 \%$ of patients in the internal medicine department had a recorded mental illness, highlighting the need for mental health support in wards that are not primarily for psychiatric care. Mental health issues are associated with chronic disease ${ }^{[2]}-$ HIV and TB specifically ${ }^{[1]}$ - suggesting that there may be patients with undiagnosed mental health issues in the sample population, which is taken from a department with a high prevalence of both NCDs and chronic infectious diseases. This is supported by a recent study in the Western Cape, where $>50 \%$ of patients with hypertension, chronic respiratory disease or diabetes mellitus showed signs of depression. ${ }^{[17]}$ Doctors should be aware of the high prevalence of comorbid mental health issues in patients with chronic diseases and how mental health issues may affect a patient's response to treatment. Mental health screening should be implemented in consultations for patients with chronic illnesses to encourage early intervention.

\section{The contribution of HIV}

Of the patients admitted to the internal medicine department, 29.7\% were diagnosed with HIV, either before admission or during their hospital stay. Less than half of these patients were currently using ARVs, an indication of the many challenges faced by national efforts to upscale HIV treatment over the past 10 years. ${ }^{[20]}$

Treatment stage was significantly associated with CD4+ count. Less than half of the patients using ARVs were classified as having a very low $\mathrm{CD} 4+$ count $(<200$ cells $/ \mu \mathrm{L})$, whereas $>70 \%$ of patients who had defaulted from ARV treatment were classified as having a very low CD4+ count (Fig. 2). Treatment stage also had significant implications for the burden of disease; patients on ARVs did not have a greater average length of stay than the sample population as a whole, but patients who had defaulted from treatment stayed on average 2.5 days longer than those on treatment, and patients who were ARV naive stayed for 1.8 days longer than those on treatment. This highlights the ongoing need for an effective HIV counselling and treatment programme at primary care level, a challenge that is still faced after aggressive government interventions over the past decade. ${ }^{[20]}$ While the patient is in hospital, adherence counselling can be helpful to try to prevent future admissions, as well as encouraging patients to go to their local ARV clinic after discharge.

\section{Study limitations and strengths}

This study had a number of limitations. The methodology involved folder review by multiple students, possibly leading to discrepancy in the recording of variables. Reliance on clinical records may have led to incorrect interpretation of doctors' handwritten notes, and no other sources were available to ensure that all handwritten notes were recorded correctly and completely. With regard to lifestyle risk factors, it is likely that the information provided by the patients was strongly affected by social stigma, particularly in the case of illicit drug use, resulting in under-reporting of these risk factors.

Selection bias was present owing to exclusion of patients who were discharged after the end of the month of May, as well as exclusion of those recorded as deceased, because of inaccessibility of folders. Although the majority of patient folders were found on the hospital's intranet database, 35 folders were inaccessible. The population sample included only one month of admissions from a single department at a district hospital, so the results cannot be generalised to other hospital departments or to other districts in SA. Owing to the cross-sectional nature of the study, no causative associations can be drawn between lifestyle factors and comorbidities, or between various comorbidities.

Strengths include the large sample size $(N=491)$ and the use of primary sources of patient information. Access to clinical records meant that there was no reliance on self-reporting of medical conditions.

\section{Conclusions}

This study described multimorbidity and factors contributing to the length of stay of patients in a district hospital. Multimorbidity was found to be the norm among patients, which should encourage healthcare professionals to shift from the view of a single diagnosis with comorbidities, to a single case of multimorbidity.

Multimorbidity has several facets, including biological interactions between diseases, risk factors and treatments; patient perspectives; health worker perspectives; and implications for health systems. ${ }^{[19]}$ An understanding of the patient perspective can be beneficial, where according to Shippee's model ${ }^{[18]}$ patient workload and capacity interact to affect healthcare utilisation and health outcomes.

An increased length of stay was found to be associated with HIV and/or TB diagnosis, non-adherence to ARV treatment or ARV naivety, and alcohol and drug use. This is a reflection of health challenges at a community and primary level of care, and these factors cannot be impacted upon by the district hospital staff in isolation. To improve the health of communities, we require partnerships between doctors, community health providers and patients with their families. Good communication is essential between levels of care.

The study findings have significant research implications. An understanding of multimorbidity can be used when designing medical education programmes, clinical drug trials and treatment regimens as well as when reforming health systems.

Acknowledgements. The authors thank Dr Jim te Water Naude for his contribution to the conceptualisation of the article, and Raphaela Böhmer, Emily Street and Danielle Williams for data capturing.

Author contributions. EdV conceptualised the research, SR collected data, both authors participated in the analysis and interpretation of data, SR drafted the manuscript, both authors revised the manuscript, and EdV finalised the article. Both authors contributed to the intellectual content of the article, and both read and approved the final manuscript.

Funding. None.

Conflicts of interest. None.

1. Mayosi BM, Flisher AJ, Lalloo UG, Sitas F, Tollman SM, Bradshaw D. The burden of noncommunicable diseases in South Africa. Lancet 2009;374(9692):934-947. https://doi.org/10.1016 S0140-6736(09)61087-4

2. Vos T, Barber RM, Bell B, et al. Global, regional, and national incidence, prevalence, and years lived with disability for 301 acute and chronic diseases and injuries in 188 countries, $1990-2013$ A systematic analysis for the Global Burden of Disease Study 2013. Lancet 2015;386(9995):743-800 https://doi.org/10.1016/S0140-6736(15)60692-4 
3. Kevany S, Meintjes G, Rebe K, Maartens G, Cleary S. Clinical and financial burdens of secondary level care in a public sector antiretroviral roll-out setting (G F Jooste Hospital). S Afr Med J 2009;99(5):320-325. Care in a public sector antiretroviral roll-out setting (G F Jooste Hospital). S Afr Med ) 2009;99(5):320-325.
4. Oni T, Unwin N. Why the communicable/non-communicable disease dichotomy is problematic for

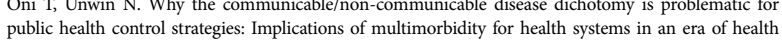
public health control strategies: Implications of multimorbidity for health systems
transition. Int Health 2015;7(6):390-399. https://doi.org/10.1093/inthealth/ihv040

5. Clark SJ, Gómez-Olivé FX, Houle B, et al. Cardiometabolic disease risk and HIV status in rural South Clark SJ, Gómez-Olivé FX, Houle B, et al. Cardiometabolic disease risk and HIV status in rural South
Africa: Establishing a baseline. BMC Public Health 2015;15:135. https://doi.org/10.1186/s12889-015Africa:

6. Oni T, Youngblood E, Boulle A, McGrath N, Wilkinson RJ, Levitt NS. Patterns of HIV, TB, and noncommunicable disease multimorbidity in peri-urban South Africa - a cross sectional study. BMC Infect Dis 2015;15:20. https://doi.org/10.1186/s12879-015-0750-1

7. Di Cesare M, Khang YH, Asaria P, et al. Inequalities in non-communicable diseases and effective responses. Lancet 2013;381(9866):585-597. https://doi.org/10.1016/S0140-6736(12)61851-0

8. Groenewald P, Evans J, Morden E, et al. Western Cape Mortality Profile 2012. Cape Town: South African Medical Research Council, 2015.

9. Barnett K, Mercer SW, Norbury M, Watt G, Wyke S, Guthrie B. Epidemiology of multimorbidity and implications for health care, research, and medical education: A cross-sectional study. Lancet 2012;380(9836):37-43. https://doi.org/10.1016/S0140-6736(12)60240-2

10. Boyd CM, Martin Fortin MD. Future of multimorbidity research: How should understanding of multimorbidity inform health system design? Public Health Rev 2010;32(2):451-474. https://doi. multimorbidity inform

11. Wallis LA, Gottschalk SB, Wood D, Bruijns S, De Vries S, Balfour C, Cape Triage Group. The Cape Triage Score - a triage system for South Africa. S Afr Med J 2006;96(1):53-56.

12. Western Cape Department of Health. Healthcare 2030: The Road to Wellness. Cape Town: Western Cape Department of Health, 2014:37. https://www.westerncape.gov.za/assets/departments/health/ healthcare2030.pdf (accessed 12 May 2017).
13. Everett-Murphy K, Mash B, Malan Z. Helping people change: The busy healthcare practitioner's guide to providing brief behaviour change counselling on non-communicable disease lifestyle risk factors.
2014. http://www.ichange4health.co.za/wp-content/uploads/2016/01/HELPING-PEOPLE-CHANGE. pdf (accessed 12 May 2017)

14. Massyn N, Day C, Peer N, Padarath A, Barron P, English R, eds. District Health Barometer 2013/14. 4. Massyn N, Day C, Peer N, Padarath A, Barron P, English R, eds. District Health Barometer 2013/14.
Durban: Health Systems Trust, 2014. https://www.health-e.org.za/wp-content/uploads/2014/10/ Durban: Health Systems Trust, 2014.

15. Joint United Nations Programme on HIV and AIDS (UNAIDS). 90-90-90: An Ambitious Treatment Target to Help End the AIDS Epidemic. Geneva: UNAIDS, 2014.

16. Alaba O, Chola L. The social determinants of multimorbidity in South Africa. Int I Equity Health 2013;12:63. https://doi.org/10.1186/1475-9276-12-63

17. Folb N, Timmerman V, Levitt NS, et al. Multimorbidity, control and treatment of non-communicable diseases among primary healthcare attenders in the Western Cape, South Africa. S Afr Med J 2015;105(8):642-647. https://doi.org/10.7196/SAMInew.8794

18. Shippee ND, Shah ND, May CR, Mair FS, Montori VM. Cumulative complexity: A functional, patient-centered model of patient complexity can improve research and practice. J Clin Epidemiol 2012;65(10):1041-1051. https://doi.org/10.1016/j.jclinepi.2012.05.005

19. Oni T, McGrath N, BeLue R, et al. Chronic diseases and multimorbidity - a conceptual modification to the WHO ICCC model for countries in health transition. BMC Public Health 2014;14(1):575. https://doi. org/10.1186/1471-2458-14-575

20. Mayosi BM, Lawn JE, van Niekerk A, Bradshaw D, Karim SS, Coovadia HM, Lancet South Africa team. Health in South Africa: Changes and challenges since 2009. Lancet 2015;380(9858):2029-2043. https:// doi.org/10.1016/S0140-6736(12)61814-5

Accepted 13 July 2017 